\title{
Secreted frizzled-related protein 2 prevents pressure-overload-induced cardiac hypertrophy by targeting the Wnt/ $\beta$-catenin pathway
}

\author{
Wen-Ying Wei ${ }^{3} \cdot$ Qing Zhao $^{1} \cdot$ Wen-zhong Zhang ${ }^{1} \cdot$ Mao-jing Wang ${ }^{1} \cdot$ Yan Li $^{1} \cdot$ Shi-zhong Wang ${ }^{2} \cdot$ Ning Zhang $^{1}$ (i)
}

Received: 15 November 2019 / Accepted: 18 June 2020 / Published online: 6 July 2020

(c) Springer Science+Business Media, LLC, part of Springer Nature 2020

\begin{abstract}
Background and aim Secreted frizzled-related protein 2 (sFRP2) has been reported to be involved in cardiovascular diseases. However, its role in cardiac hypertrophy induced by pressure overload is still elusive. We aimed to examine the role of sFRP2 in the development of cardiac hypertrophy in vivo and in vitro.

Methods and results Following cardiac hypertrophy stimulated by aortic banding (AB), the expression of sFRP2 was downregulated in the hypertrophic ventricle. Adeno-associated virus 9 (AAV9) was injected through the tail vein to overexpress sFRP2 in the mouse myocardium. Overexpression of sFRP2 alleviated cardiomyocyte hypertrophy and interstitial fibrosis, as identified by the reduced cardiomyocyte cross-sectional area, heart weight/body weight ratio, and left ventricular (LV) collagen ratio. Additionally, sFRP2 decreased cardiomyocyte apoptosis induced by pressure overload. Western blot showed that sFRP2 prevented the expression of active $\beta$-catenin. The $\mathrm{Wnt} / \beta$-catenin agonist $\mathrm{LiCl}(1 \mathrm{mmol} / \mathrm{kg})$ abolished the inhibitory effects of sFRP 2 on cardiac hypertrophy and apoptosis, as evidenced by the increased cross-sectional area and LV collagen ratio and the deterioration of echocardiographic data.

Conclusion Our study indicated that decreased sFRP2 levels were observed in failing mouse hearts. Overexpression of sFRP2 attenuated myocyte hypertrophy and interstitial fibrosis induced by hypertrophic stimuli by inhibiting the Wnt/ $\beta$ catenin pathway. We revealed that SFRP2 may be a promising therapeutic target for the development of cardiac remodeling.
\end{abstract}

Keywords Secreted frizzled-related protein $2(\mathrm{sFRP} 2) \cdot$ Cardiac hypertrophy $\cdot$ Fibrosis $\cdot$ Apoptosis $\cdot$ Pressure overload

\section{Introduction}

Heart failure and its underlying cardiac hypertrophy processes are spreading worldwide [1]. Cardiac hypertrophy is identified as compensatory pathological changes in response

Electronic supplementary material The online version of this article (https://doi.org/10.1007/s11010-020-03802-x) contains supplementary material, which is available to authorized users.

Ning Zhang

zhangningqdfy@yeah.net

1 Department of Cardiology, The Affiliated Hospital of Qingdao University, No. 16 Jiangsu Road, Qingdao 266000, People's Republic of China

2 Department of Cardiovascular Surgery, The Affiliated Hospital of Qingdao University, Qingdao, China

3 Department of Intensive Care Unit, The Affiliated Hospital of Qingdao University, Qingdao, China to chronic arterial hypertension and/or aortic valve stenosis. Characterized by the deposition of cardiac interstitial collagen and cardiomyocyte enlargement, cardiac hypertrophy is regarded as a major predictor for the development of heart failure. Hence, inhibition of pathological cardiac hypertrophy can serve as an effective therapeutic strategy for curing heart failure.

The Wnt/ $\beta$-catenin pathway is evolutionarily conserved throughout metazoans and participates in various developmental processes including early cardiomyogenesis [2]. Wnt/ $\beta$-catenin signaling is usually silent in adult organs, but reactivates after acute injury. Previous studies have found that $\mathrm{Wnt} / \beta$-catenin signaling is closely related to the mediation of cardiomyocyte enlargement and apoptosis. It has been demonstrated that increased Wnt3a expression activated caspases and aggravated myocyte apoptosis induced by hypoxia reoxygenation [3]. Furthermore, activation of Wnt/ $\beta$-catenin is observed in cardiac and renal tissues following pressure overload stimulation, and blockage of the signaling 
attenuates chronic organ remodeling in the heart and kidney [4]. Secreted frizzled-related protein 2 (sFRP2), as a soluble protein, directly interacts with Wnt extracellularly and prevents its interaction with frizzled; this is involved in the regulation of $\mathrm{Wnt} / \beta$-catenin signaling. Lin et al. found that SFRP2 was downregulated after cardiac infarction in mice and elaborated that SFRP2 may be an antifibrotic target by regulating cardiac fibroblast (CF) growth and extracellular matrix remodeling [5]. Nevertheless, whether sFRP2 can affect pressure-overload-induced cardiac hypertrophy through Wnt/ $\beta$-catenin signaling remains elusive.

In the present study, we explored the role of sFRP2 in the pathogenesis of cardiac hypertrophy induced by aortic banding $(\mathrm{AB})$, as well as the underlying mechanism.

\section{Material and methods}

\section{Animals and reagents}

C57BL/6 male mice (4 weeks) were obtained from the Institute of Laboratory Animal Science, CAMS \& PUMC (Beijing, China). Antibodies against $\beta$-catenin (ab32572), total-GSK-3 $\beta$ (ab32391), phospho-GSK-3 $\beta$ (ab75814), BAX (ab32503), Bcl-2 (ab196495), cleaved Caspase-3 (ab214430), and GAPDH (ab181602) were purchased from Abcam. Active $\beta$-catenin $(19,807)$ was obtained from Cell Signaling Technology and angiotensin (Ang II, A9525) and lithium chloride $(\mathrm{LiCl}, 746,460)$ were purchased from Sigma-Aldrich. Recombinant adeno-associated viruses of serotype 9 (AAV9) were produced and purchased from Hanbio Biotechnology Co. (Shanghai, China). AAV9 vectors were packaged with single-stranded DNA containing enhanced green fluorescent protein (GFP) gene or sFRP2, which was driven by the human cytomegalovirus (CMV) promoter (AAV9-CMV-GFP or AAV9-CMV-GFP-sFRP2). Vector titers were determined by using qPCR with primers against the CMV promoter region. Recombinant sFRP2 (1169-FR) was obtained from R\&D Systems.

\section{Animal experiments}

All animal procedures were by approved the Guidelines for the Care and Use of Laboratory Animals published by the United States National Institutes of Health (NIH Publication, revised 2011) and the institutional guidelines of the Animal Care and Use Committee of Affiliated Hospital of Qingdao University (Qingdao, China). All eighty male C57BL/6 mice were housed and drank freely under specific pathogen-free conditions under a 12-h light/12-h dark cycle and fed standard chow diet (13.5 kcal\% fat, $32.98 \mathrm{kcal} \%$ Protein, $56.7 \mathrm{kcal} \%$ Carbohydrate). AAV9-CMV-sFRP2 or AAV9-CMV-GFP $\left(1 \times 10^{12} \mathrm{drp}\right)$ was injected through the tail vein to overexpress sFRP2 in the mouse myocardium. Four weeks later, C57BL/6 mice were anesthetized by intraperitoneal injection of $3 \%$ sodium pentobarbital and placed on a ventilator. The $\mathrm{AB}$ and sham operation were performed in $24-26 \mathrm{~g}$ male $\mathrm{C} 57 \mathrm{Bl} / 6 \mathrm{~J}$ mice ( 8 weeks old) according to a previous study [6]. Additionally, adequate aortic banding was confirmed by Doppler echocardiogram. The sham mice underwent a similar operation without aorta constriction. $\mathrm{LiCl}$, a Wnt//-catenin signaling agonist, was administered by intragastric administration via a $1 \mathrm{mmol} / \mathrm{kg}$ dose once a day and maintained for 2 weeks.

\section{Echocardiography and hemodynamic measurements}

Mouse echocardiography was performed under sedation by $1.5 \%$ isoflurane inhalation. Short-axis parasternal views of the left ventricular systolic and diastolic parameters were obtained immediately at the mid-papillary level using a Vivid echocardiography apparatus with a $10-\mathrm{MHz}$ probe (Esaote SpA, Genoa, Italy). M-mode measurements of the magnitude of the LV walls and chamber were achieved by two-dimensional guidance from the left ventricular shortaxis view in a blinded manner as described previously [6, 7]. LV end-diastolic diameter (LVEDd), LV end-systolic diameter (LVESd), and end-diastolic interventricular septal thickness (IVSd) were determined. The LV fractional shortening (FS) was computed using the following formula: FS $(\%)=($ LVEDd-LVESd $) /$ LVEDd $\times 100$.

For invasive hemodynamic analysis, mice were primarily anesthetized with $1.5 \%$ isoflurane inhalation. An incision was subsequently made in the right carotid artery and pressure-volume (PV) loop measurements were performed using a 1.4 Fr micromanometer conductance catheter (Millar, Houston, USA). Hemodynamic data were collected at steady state; PV loop parameters including the maximum rate of pressure development $(\mathrm{d} p / \mathrm{d} t \max )$ and minimum rate of pressure decay $(\mathrm{d} p / \mathrm{d} t \mathrm{~min})$ were calculated using the PVAN data analysis software (Millar, Houston, USA).

\section{Histological measurements}

The harvested heart was fixed in $4 \%$ paraformaldehyde for $24 \mathrm{~h}$, subsequently dehydrated in ethanol and xylene series, and finally embedded in paraffin. To evaluate the cross-sectional sizes of cardiomyocytes and cardiac interstitial fibrosis, 5- $\mu \mathrm{m}$ cardiac tissue slides were stained with hematoxylin and eosin (H\&E) and picrosirius red (PSR). Subsequently, images of the slides were obtained by optical microscopy with a Nikon Photo-Imaging System (H550L, Tokyo, Japan). For cardiomyocytes sizes and interstitial fibrosis quantification, images were analyzed with the Image-pro Plus 6.0 software (Maryland, USA). 
For immunohistochemistry of myocardial sFRP2 expression, the cardiac tissue sections were deparaffinized, blocked with $8 \%$ goat serum, and then incubated with 1:100 diluted anti-sFRP2 antibody overnight at $4{ }^{\circ} \mathrm{C}$. After washing, the slides were incubated for $1 \mathrm{~h}$ with anti-rabbit HRP reagent (Gene Tech, Shanghai, China) at $37{ }^{\circ} \mathrm{C}$, rinsed, and developed with a peroxide-based substrate DAB kit for $5 \mathrm{~min}$ (Gene Tech, Shanghai, China). Finally, images of the slides were obtained by light microscopy with a Nikon PhotoImaging System (H550L, Tokyo, Japan).

\section{TUNEL assay}

Apoptosis was detected in the cardiac tissues using the Apoptosis Assay Kit (Millipore, Temecula, CA) according to the manufacturer's instructions. The myocardial tissue sections were deparaffinized and preconditioned with proteinase $\mathrm{K}(20 \mu \mathrm{g} / \mathrm{ml})$, after which they were incubated with fluorescein-labeled dUTP. Quantitation of apoptotic cells, as shown by nuclear fragmentation and chromatin condensation, was conducted with the Image-pro Plus 6.0 software.

\section{Neonatal rat cardiomyocyte (NRCM) isolation and determination of myocyte surface area}

NRCMs were isolated from 1- to 3-day-old Sprague-Dawley (SD) rats as shown previously [6]. Briefly, twenty neonatal rat ventricles were minced into $1 \mathrm{~mm}$ small fragments and digested with $0.25 \mathrm{~g} / \mathrm{l}$ trypsin and $5 \mathrm{~g} / \mathrm{l}$ collagenase at $37{ }^{\circ} \mathrm{C}$ six times for $10 \mathrm{~min}$. The resultant suspension was collected by centrifugation at $600 \mathrm{~g}$ for $10 \mathrm{~min}$ and strained through a $70-\mu \mathrm{m}$ filter. Then, the cell suspension was reseeded into fresh DMEM/F12 medium containing 10\% (V/V) fetal bovine serum (FBS) (Ausbian, Australia) for $1.5 \mathrm{~h}$. Afterwards, the cardiac fibroblasts were abandoned and the extracted cardiomyocytes were transferred to a new plate and cultured accordingly. For in vitro experiments, cardiomyocytes were prepared as quiescent by serum starvation for $12 \mathrm{~h}$, after which the cells were incubated with $\mathrm{LiCl}$ $(2.5 \mathrm{mM}, 12 \mathrm{~h})$ followed by Ang II $(1 \mu \mathrm{M})$ stimulation for the indicated amount of time.

For evaluation of the myocyte surface area, cardiomyocytes were seeded on coverslips and incubated with $15 \mathrm{nM}$ sFRP2 and/or not $2.5 \mathrm{mM} \mathrm{LiCl}$ following stimulation with Ang II for $24 \mathrm{~h}$. To block the $\mathrm{Wnt} / \beta$-catenin signaling, NRCMs were pretreated with $\mathrm{LiCl}$ for $12 \mathrm{~h}$ followed by Ang II stimulation. The coverslips were washed twice with icecold PBS and fixed with PBS plus 4\% paraformaldehyde for $30 \mathrm{~min}$. The myocytes were permeabilized with PBS containing $0.1 \%$ Triton $\mathrm{X}$ for $10 \mathrm{~min}$ and then blocked with $2 \%$ BSA in PBS for $1 \mathrm{~h}$. Cardiomyocytes were incubated with primary antibody against $\alpha$-actin (Abcam, Cambridge, MA) overnight at $4{ }^{\circ} \mathrm{C}$ and reacted with a fluorescent secondary antibody. For determination of the myocyte surface area, fluorescent images were captured by an inverted fluorescence microscope and calculated using the Image-pro Plus 6.0 software.

\section{Quantitative real-time reverse transcriptase-polymerase chain reaction (RT-PCR)}

LV myocardial tissues were immediately dissected and total RNA was extracted from mouse cardiac tissues and cultured cardiomyocytes using the TRIzol reagent (Invitrogen, Carlsbad, CA, USA). First strand cDNA was made using the cDNA reverse transcription kit (Roche, Mannheim, Germany). Quantification of specific gene expression was performed using the LightCycler 480 SYBR Green Master Mix (Roche, Mannheim, Germany) and mRNA sequencespecific PCR primers were designed using Primer Express software; the primer sequences are shown in Supplementary Table 1. Gene expression of the housekeeping gene GAPDH was utilized as a relative quantitative reference.

\section{Immunoblot analysis}

Cardiac tissues and cultured NRCMs were homogenized in RIPA lysis buffer, and the concentration of the protein lysates was determined by a BCA assay kit as indicated previously [6]. Protein extracts were separated by $10 \%$ SDS polyacrylamide gels under denaturing conditions and blotted onto polyvinylidene difluoride (PVDF) membranes. PVDF membranes were blocked with $5 \%$ bovine serum albumin/ TBST and then incubated with the following primary antibodies: $\beta$-catenin, active $\beta$-catenin, phospho-GSK-3 $\beta$, totalGSK-3 $\beta$, BAX, BCL-2, and cleaved-caspase 3 diluted in $5 \%$ bovine serum albumin, followed by incubation with the appropriate conjugated anti-rabbit or anti-mouse secondary antibodies (Cell Signaling Technology). Signals were obtained using a two-color infrared imaging system (Odyssey; LI-COR Biosciences, Lincoln, NE, USA). Blot quantification was performed with the ImageJ software (NIH, Bethesda, MD, USA) and normalized to GAPDH protein expression.

\section{Statistical analyses}

Data were presented as the mean \pm SD. Differences between groups were analyzed with one-way analysis of variance (ANOVA) using GraphPad Prism 6 (Graphpad Software Inc, La Jolla, CA). Comparisons of two unpaired groups utilized the two-sided Student's $t$ tests. $P \leq 0.05$ was considered statistically significant. 


\section{Results}

\section{sFRP2 expression was downregulated in cardiac hypertrophy in vivo and in vitro}

We detected the expression of sFRP2 in C57BL/6 mice for 1,2 , and 4 weeks after $A B$ operation. Our data showed that sFRP2 $\mathrm{mRNA}$ and protein expression was increased in the 1st week after $\mathrm{AB}$ operation and subsequently decreased during the 4th week (Fig. 1a-b). NRCMs were cultured in vitro and stimulated with $1 \mu \mathrm{M}$ Ang II for $24 \mathrm{~h}$. Western blotting results indicated that sFRP2 expression was significantly decreased in Ang II-treated NRCMs compared with the PBS-treated group (Fig. 1c). These results suggested that the changeable expression of sFRP2 may have possible roles in $\mathrm{AB}$-induced cardiac hypertrophy.

\section{Overexpression of sFRP2 attenuated pressure-overload-induced cardiac hypertrophy}

To examine the effects of sFRP2 on AB-induced cardiac hypertrophy and accompanying dysfunction, we specifically overexpressed sFRP2 in the myocardium by injecting AAV9 encoding sFRP2 into the tail vein of mice [8]. We observed that compared with baseline levels, cardiac sFRP2 expression was increased by approximately 3.5 fold in AAV9-sFRP2-infected mice after 8 weeks (Fig. 2a-c). To investigate the potential protective role of sFRP2 in the development of pressure-overload-induced cardiac hypertrophy, $\mathrm{AB}$ and sham operations were performed 4 weeks after AAV9-sFRP2 or AAV9-GFP injection. We detected mRNA expression of fetal gene BNP in hypertrophic hearts. Our results demonstrated that $\mathrm{sFRP} 2$ overexpression retarded the development of cardiac hypertrophy and improved the deteriorative cardiac functions in AB-treated mice, as indicated by their reduced heart weight/body weight (HW/BW) ratio and decreased BNP mRNA expression (Fig. 2e-f). Four weeks after $\mathrm{AB}$ operation, $\mathrm{H} \& \mathrm{E}$ staining was used to evaluate the cross-sectional area of cardiomyocytes. The results showed that cardiomyocyte cross-sectional areas were decreased in AAV9-sFRP2-treated mice after AB insult compared with AAV9-GFP-treated mice (Fig. 2g). The echocardiographic and hemodynamic measurements reflected the anti-hypertrophy and cardiac function protective effects of overexpressed sFRP2, which presented as a reduction in LVEDd, LVEDd, and IVSd and increase in $\mathrm{dp} /$ $\mathrm{dt}$ max, dp/dt min, and FS in mice subjected to AB surgery (Fig. 2h). These data suggested that the protective role of sFRP2 was achieved in response to hypertrophic insults.

\section{SFRP2 overexpression retarded pressure-overload-induced cardiac fibrosis}

To evaluate the role of sFRP2 in cardiac fibrosis, PSR staining was performed. As identified by the decreased interstitial fibrosis ratio (Fig. 2i, k), overexpression of sFRP2 markedly mitigated myocardial fibrosis induced by hypertrophic stimuli. Meanwhile, the induction of fibrotic markers including collagen $\mathrm{I} \alpha$, collagen III $\alpha$, and periostin by $\mathrm{AB}$ were markedly increased in hypertrophic mice; sFRP2 significantly blunted collagen I $\alpha$, collagen III $\alpha$, and periostin mRNA expression (Fig. 2k) in hypertrophic hearts, whereas there were no statistically significant differences between the sham-sFRP2- vs. sham-GFP-treated mice. These results implied that sFRP2 plays a role in mitigating cardiac fibrosis under pressure overload.

\section{SFRP2 overexpression blunted AB-induced cardiomyocyte apoptosis and the $\mathrm{Wnt} / \boldsymbol{\beta}$-catenin pathway}

We further investigated the effects of sFRP2 on cardiac apoptosis. TUNEL staining was performed in cardiac
A

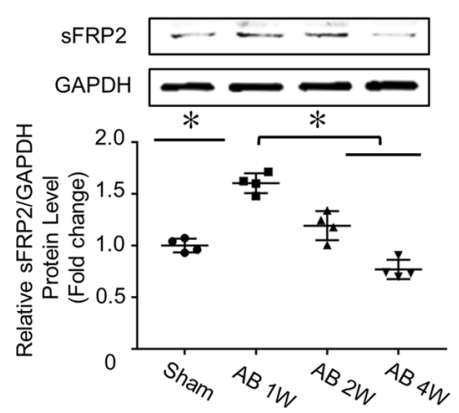

B

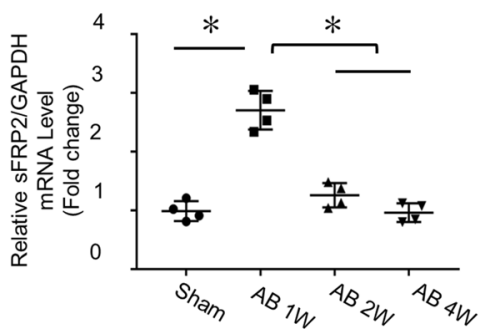

C

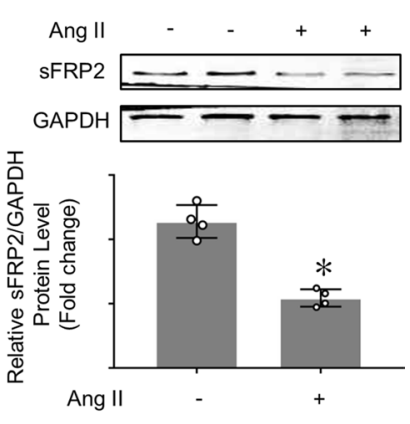

Fig. 1 sFRP2 expression is downregulated in failing heart tissue stimulated by pressure overload. a and $b$ are Protein and mRNA levels of sFRP2 in samples from C57BL/6 mice at the indicated times after sham or aortic banding (AB) surgery $(n=4 ; * P<0.05)$. c Pro- tein expression of sFRP2 in neonatal rat cardiomyocytes (NRCMs) following Ang II stimulation for $24 \mathrm{~h}$. $(n=3$ repetitions; $* P<0.05)$. Data are presented as the mean \pm SD. $* P<0.05$ 
tissue sections. Our results indicated that a large number of TUNEL positive nucleus were present in the hypertrophic cardiac slices, whereas positive nucleus signal was significantly reduced in the sFRP2-overexpressing hearts (Fig. 3a, b). Protein levels of cleaved-caspase 3, BAX, and BCL-2 were assessed by Western blotting and indicated that overexpressed sFRP2 markedly decreased the expression of the pro-apoptosis proteins cleaved-caspase 3 and BAX and increased the expression of anti-apoptosis proteins BCL-2 (Fig. 3c).

A previous study revealed that sFRP2 can potently blunt canonical $\mathrm{Wnt} / \beta$-catenin signaling in epithelial ovarian cancer [9], while in our present study, pressure overload induced by $\mathrm{AB}$ could also induce the activation of this canonical Wnt//-catenin signaling (Fig. 3d). More importantly, overexpression of sFRP2 dramatically inhibited the $\mathrm{Wnt} / \beta$-catenin pathway, indicated by the decreased expression of $\beta$-catenin and active $\beta$-catenin (Fig. $3 \mathrm{~d}$ ). Collectively, these results suggested a cardioprotective role for sFRP2 that reduces cardiomyocyte apoptosis via inhibition of the $\mathrm{Wnt} / \beta$-catenin pathway.

\section{sFRP2 alleviated Ang II-induced cardiomyocyte hypertrophy and apoptosis via suppressing the Wnt/ $\beta$-catenin pathway}

Since Ang II stimulation resulted in a remarkable downregulation of sFRP2 expression (Fig. 1c), a subsequent study was performed to evaluate the specific molecular mechanisms of sFRP2 in Ang II-induced myocyte hypertrophy. NRCMs were isolated and stimulated by Ang II $(1 \mu \mathrm{M})$ for $24 \mathrm{~h}$ to establish cardiomyocyte hypertrophy model in vitro. As shown in Fig. 3e-g, sFRP2 mitigated Ang II-induced cardiomyocyte hypertrophy, which was indicated by a smaller cell surface area and decreased BNP mRNA expression compared with the positive control group. Additionally, sFRP2 alleviated the vast apoptosis of cardiomyocytes induced by Ang II (Fig. 3h).

To examine whether sFRP2 regulates Ang II-induced cardiac hypertrophy and apoptosis in a Wnt/ $\beta$-catenin signaling-mediated way, NRCMs were pretreated with $2.5 \mathrm{mM}$ $\mathrm{LiCl}$ (a Wnt/ $\beta$-catenin signaling agonist) for $12 \mathrm{~h}$ followed by Ang II for $24 \mathrm{~h}$. Thereafter, the role of $\mathrm{LiCl}$ in SFRP2 coincubated with AngII stimulation was evaluated. Our data showed that preincubation with $\mathrm{LiCl}$ reversed the downregulation of $\beta$-catenin expression and increased levels of cleaved-caspase3 (Fig. 3h). Moreover, $\mathrm{LiCl}$ abolished the protective role of sFRP2 followed by Ang II stimulation, as evidenced by the larger cell surface area (Fig. 3e) and increased BNP mRNA expression (Fig. 3g). The results demonstrated that sFRP2 mitigated Ang II-induced cardiac hypertrophy and apoptosis, mainly by inhibiting the $\mathrm{Wnt} / \beta$ catenin pathway.

\section{sFRP2 exerted protective effects followed by pressure overload stimulation depending on the inhibition of $\mathrm{Wnt} / \boldsymbol{\beta}$-catenin signaling}

To validate the critical effects of sFRP2 overexpression on $\mathrm{Wnt} / \beta$-catenin signaling in mouse cardiac hypertrophy, $\mathrm{LiCl}(1 \mathrm{mmol} / \mathrm{kg}$ per day) was intraperitoneally injected into cardiac hypertrophy mice two weeks before sacrifice. Our results showed that $\mathrm{LiCl}$ abolished the anti-hypertrophic role of sFRP2, as identified by the increased cardiomyocyte sectional areas (Fig. 4a-b), HW/BW ratios, and BNP mRNA expression (Fig. 4d-e). Additionally, compared with the AB + AAV9-sFRP2 group, mice in the AB + AAV9sFRP2 $+\mathrm{LiCl}$ group presented more collagen deposition in the myocardium (Fig. 4a, c). Echocardiographic data indicated that $\mathrm{LiCl}$ abrogated the alleviative development of cardiac hypertrophy induced by AB following sFRP2 overexpression, which manifested as similar LVEF (\%), LVEDd, and LVESd levels between the AB + AAV9-GFP and $\mathrm{AB}+\mathrm{AAV} 9-\mathrm{sFRP} 2+\mathrm{LiCl}$ groups (Fig. $4 \mathrm{f}-\mathrm{g}$ ). Taken together, these results revealed the protection of sFRP2 against cardiac hypertrophy and fibrosis depending on the blockage of the Wnt/ $\beta$-catenin signaling pathway.

\section{Discussion}

Overarching conclusions from previous studies indicated a critical role for the dysregulated $\mathrm{Wnt} / \beta$-catenin pathway in the pathogenesis of a hypertrophic heart $[10,11]$. Inhibition of the $\mathrm{Wnt} / \beta$-catenin pathway exerted a beneficial role and resulted in improved cardiac function following ischemic insults [12]. Our study was focused on sFRP2, which is similar to Frizzled, but lacks transmembrane domains [11]. sFRP2, a novel secreted factor, is considered a multifaceted regulator of the $\mathrm{Wnt} / \beta$-catenin pathway. A previous study demonstrated that sFRP2 expression in the failing heart was increased in a $\delta$-sarcoglycan-null TO2 strain hamster [13]; however, we found that SFRP2 expression in the hypertrophic heart of C57BL/6 mice was increased after 1 week and then decreased after 4 weeks. Meanwhile, reduced sFRP2 expression was also detected in NRCMs stimulated by Ang II; this discrepancy might be attributable to the different stages of cardiac remodeling. In the early phase of a 
A

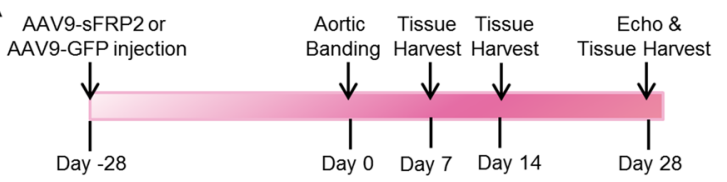

D AAV9-GFP $\frac{\text { Sham }}{\text { AAV9-sFRP2 AAV9-GFP AAV9-sFRP2 }}$

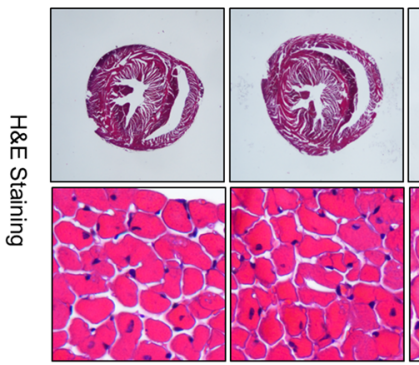

G

H
B

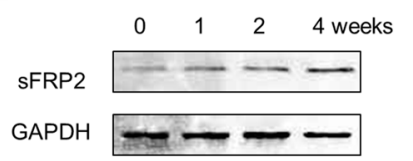

E

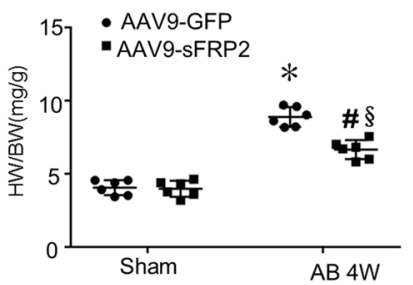

C

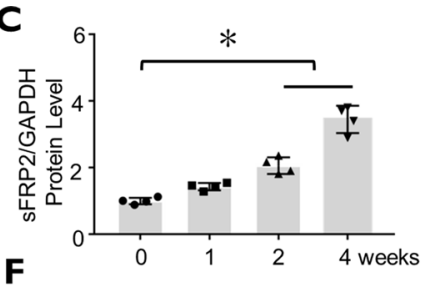

F

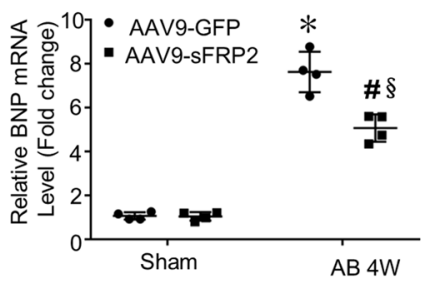

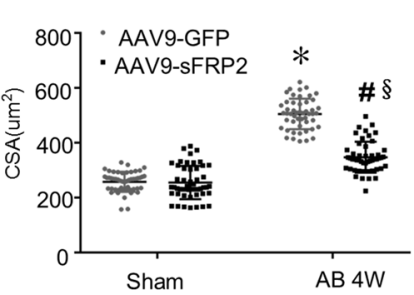

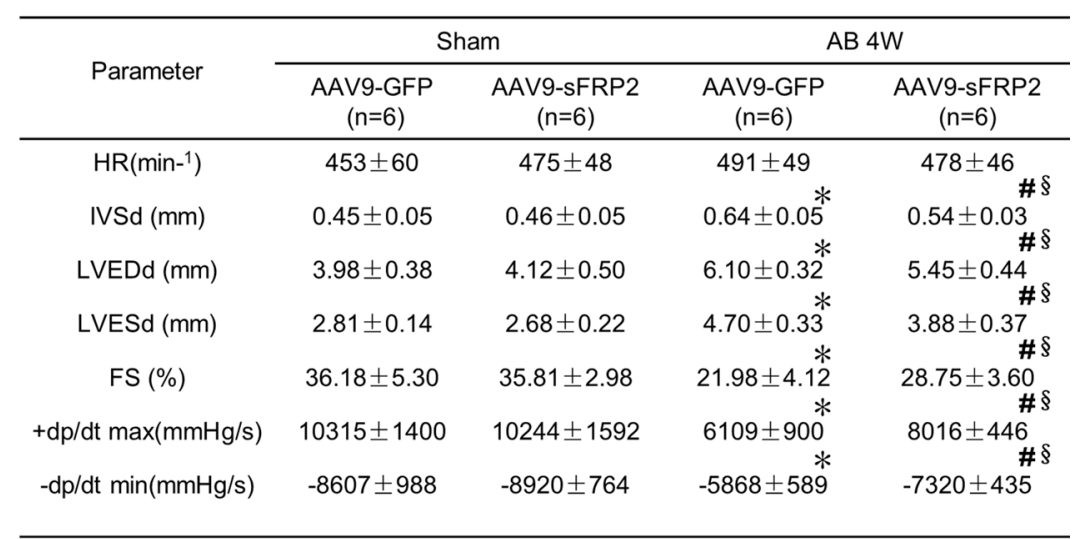

I

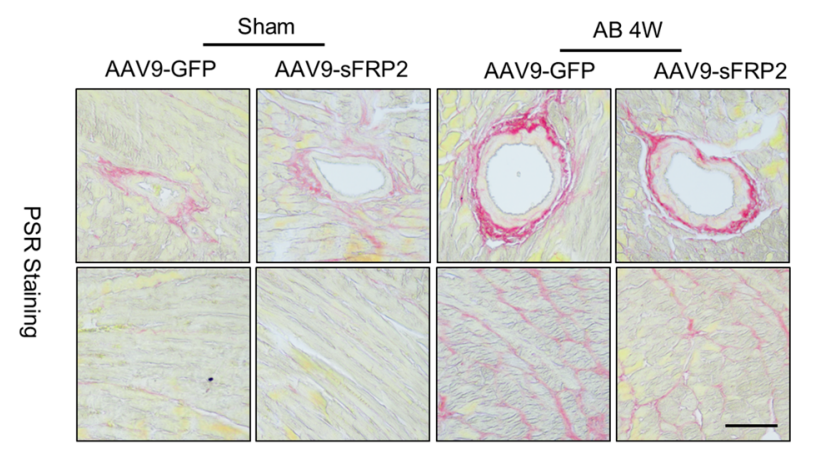

K

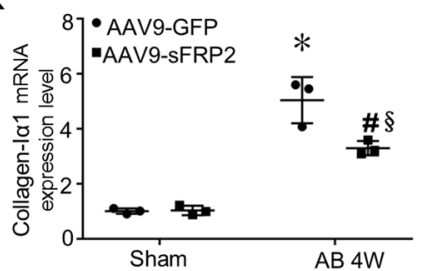

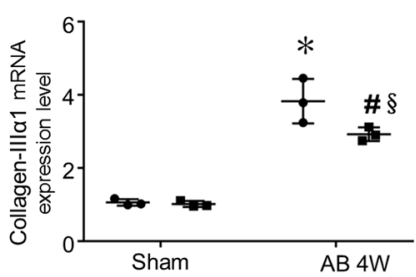

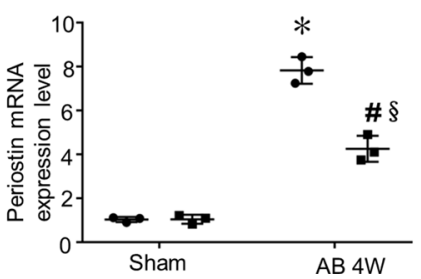


4Fig. 2 sFRP2 overexpression prevents the establishment of cardiac hypertrophy. a Schematic timeline to investigate sFRP2 overexpression effects in AB-induced left ventricular (LV) hypertrophy; $A B$ and sham operations were performed 4 weeks after AAV9-CMV-sFRP2 or AAV9-CMV-GFP injection. b and $\mathbf{c}$ Quantification of sFRP2 expression in cardiac tissues following AAV9-sFRP2 infection at the indicated times $(n=4 ; * P<0.05)$. d Four weeks after AB operation, $\mathrm{H} \& \mathrm{E}$ staining was used to evaluate the cross-sectional area of cardiomyocytes hematoxylin and eosin (H\&E) staining; characteristic images of heart cross-sections showing hypertrophic growth in the indicated mice $(n=6$ mice per group, at least 60 cells were measured per group). Bottom scale bars, $20 \mu \mathrm{m}$. e The heart weight to body weight ratio (HW/BW) in sFRP2- or GFP-overexpressing hearts 4 weeks after the sham or $\mathrm{AB}$ operation ( $n=6$ mice per group). f Real-time polymerase chain reaction (RT-PCR) analysis of brain natriuretic peptide (BNP) expression in SFRP2- or GFP-overexpressing hearts 4 weeks after the sham or $\mathrm{AB}$ operation $(n=4$ mice per group), sFRP2 overexpression retarded BNP mRNA expression in AB-treated mice. g Cardiomyocyte cross-sectional area (CSA) quantified in the sham or $\mathrm{AB}$ mice from the indicated groups $(n=6$ mice per group, at least 60 cells were measured per group). h Echocardiographic parameters including the heart rate (HR), end-diastolic interventricular septal thickness (IVSd), left ventricle end-diastolic diameter (LVEDd), left ventricle end-systolic diameter (LVESd), left ventricle fractional shortening (FS), and hemodynamic parameters, such as the maximal rate of pressure development $(\mathrm{d} p / \mathrm{d} t \max )$, maximal rate of pressure decay (dp/dt min), of each group 4 weeks after $\mathrm{AB}$ or sham surgery ( $n=6$ mice per group). i Characteristic images of picrosirius red (PSR) staining of left ventricular sections in sham and $\mathrm{AB}$ animals. $\mathbf{j}$ Quantification of interstitial fibrosis in mice from the sham and hypertrophy groups $(n=6$ mice per group, at least 12 different areas were measured per group). $\mathbf{k}$ mRNA expression of collagen I $\alpha 1$, collagen III $\alpha 1$, and periostin detected by RT-PCR analysis in each indicated group ( $n=3$ mice per group), sFRP2 significantly blunted collagen I $\alpha$, collagen III $\alpha$, and periostin mRNA expression in hypertrophic hearts. Data are presented as the mean \pm SD. $* P<0.05$ vs. the sham group, ${ }^{\#} P<0.05$ vs. the $\mathrm{AB}+\mathrm{GFP}$ group, and $\S P<0.05$ vs. the sham group.

failing heart, sFRP2 expression in cardiac tissues could be increased, which is consistent with our results that cardiac sFRP2 levels increased 1 week after AB surgery. Therefore, the mechanism to mediate the expression and functional relevance of sFRP2 requires further investigation.

$\mathrm{Wnt} / \beta$-catenin signaling was activated in cardiac tissues during the progression of cardiac hypertrophy. Blankesteijn et al. observed increased Frizzled-2 expression during the development of cardiac hypertrophy induced by aortic constriction in rat [14]. The levels of Disheveled (Dvl), a downstream molecule of Frizzled in the Wnt/ $\beta$-catenin pathway, was shown to be upregulated after pressure overload stimulation in mice $[15,16]$. Moreover, overexpression of $\beta$-catenin resulted in hypertrophic growth of cardiomyocytes in a rat myocardial infarction model [17]. Moreover, cardiac-specific deletion of $\beta$-catenin alleviated the development of cardiac hypertrophy stimulated by thoracic aortic constriction [17].
Overall, studies have indicated that $\mathrm{Wnt} / \beta$-catenin mediates hypertrophic responses upon various stimuli. Notably, sFRP2 mediated the differentiation of mouse embryonic carcinoma stem cells by blocking Wnt3a and exerted a beneficial role to alleviate apoptosis during hypoxia reoxygenation in $\mathrm{H} 9 \mathrm{C} 2$ cells $[3,18]$. sFRP2 also plays a vital role in regulating the survival signal of mesenchymal stem cells in the ischemic myocardium [19]. In the present study, we utilized AAV9-sFRP2 to transfect myocardial tissue and then performed an $\mathrm{AB}$ operation to establish a cardiac hypertrophy model. Our results showed that overexpression of sFRP2 significantly alleviated pressure-overload-induced myocardial hypertrophy via inhibition of the $\mathrm{Wnt} / \beta$-catenin pathway.

Even though cardiac hypertrophy is considered as the adaptability to multiple stimuli, increased apoptosis aggravates cardiac dysfunction when the compensatory hypertrophic response exceeds its ability. Therefore, inhibition of apoptosis is a critical strategy to prevent cardiac remodeling. Many studies have revealed that increased apoptosis is associated with aberrant activation of Wnt signaling [20], and activation of $\mathrm{Wnt} / \beta$-catenin could mediate apoptosis following intestinal ischemia/reperfusion (IR) injury [21]. As depicted in Fig. 4, we proposed a cascade of pathological change in AB-mediated hypertrophic response that triggers $\mathrm{Wnt} / \beta$-catenin activation in the myocardium. sFRP2 prevented apoptosis following IR injury by blocking canonical Wnt3a [3]. In our study, sFRP2 expression was decreased after hypertrophic stimuli both in vitro and in vivo, and overexpression of sFRP2 attenuated AB-induced apoptosis by inhibiting $\mathrm{Wnt} / \beta$-catenin signaling. Moreover, our in vitro study delineated that $\mathrm{LiCl}$ abolished the anti-apoptotic role of sFRP2 in NRCMs with Ang II stimulation.

Cardiac fibrosis is another important feature in the development of cardiac remodeling and is closely associated with cardiac diastolic dysfunction. Previous studies have delineated that Wnt expression is a major downstream molecular pathway of TGF- $\beta$-induced cardiac fibrogenesis [22]. Meanwhile, sFRP2 scavenges extracellular Wnts and blocked TGF- $\beta$-mediated myofibroblast formation in autoimmune myocarditis, resulting in the alleviation of cardiac fibrosis [23]. An in vitro study showed that sFRP2 promoted $\mathrm{C} 2 \mathrm{C} 12$ myoblast proliferation, but did not reveal a change in $\alpha$-SMA expression after sFRP2 treatment [24]. In our study, sFRP2 mitigated Ang II-induced NRCMs hypertrophy. Excessive activation of $\mathrm{Wnt} / \beta$-catenin signaling is associated with abnormal cardiac fibrosis, while sFRP2 is traditionally considered a Wnt inhibitor in that it completely interacts with Frizzled receptors [25]. However, sFRP2 possessed 
A
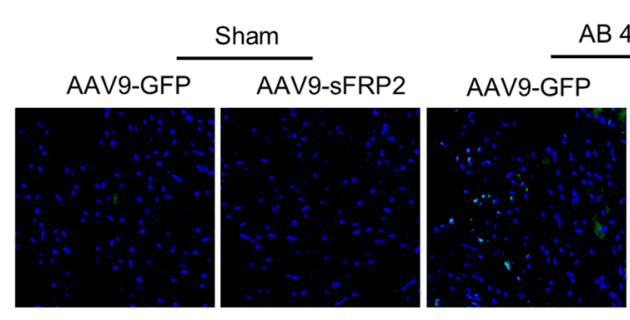

C

Cleaved-caspase3

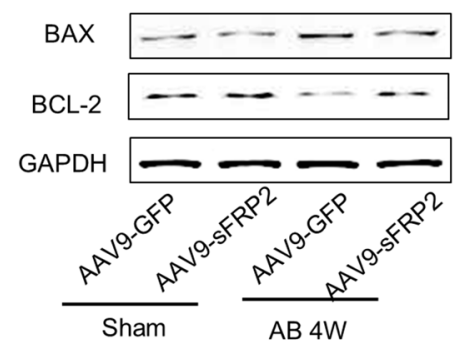

D

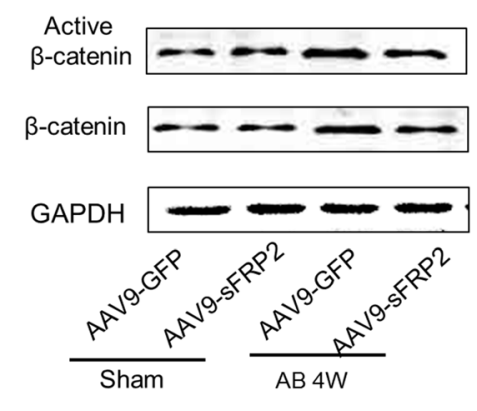

E
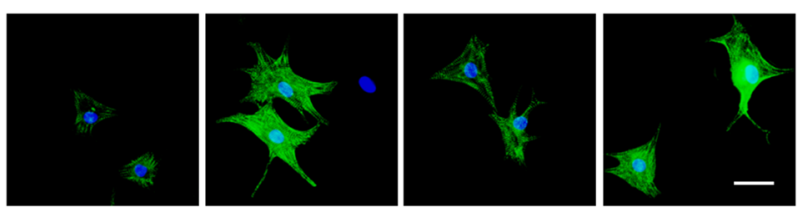

Ang II

sFRP2

$\mathrm{LiCl}$

\section{G}

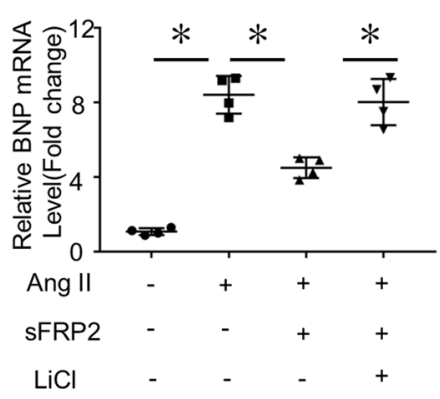

B

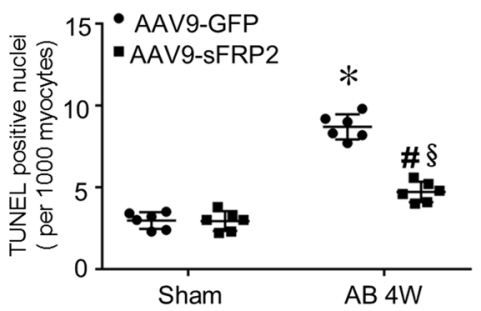

Cleaved-caspase3/ BAX/BCL-2 Ratio GAPDH
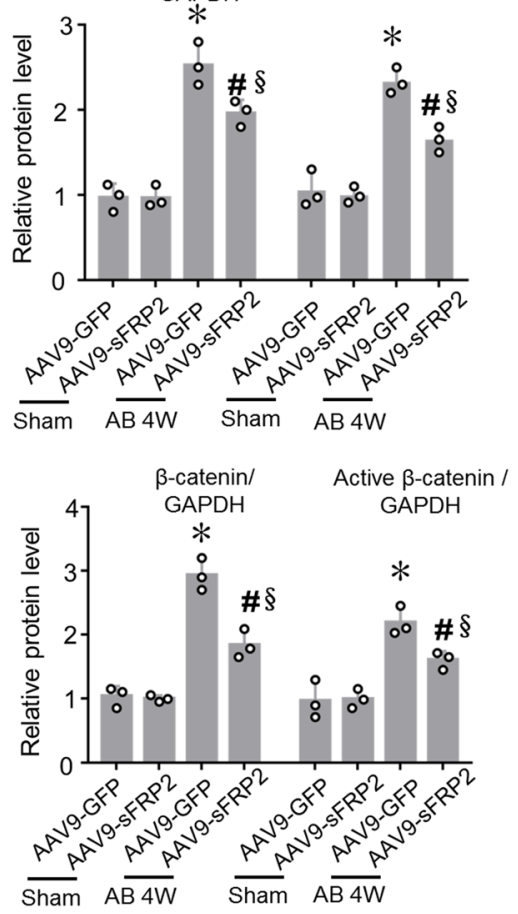

\section{H}

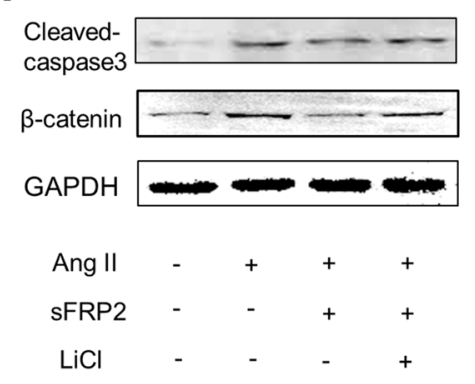

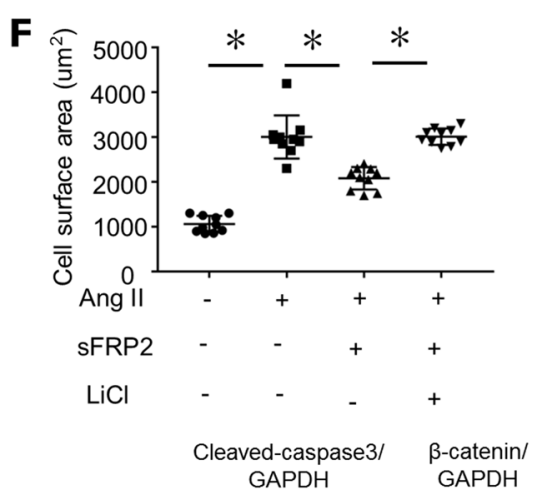

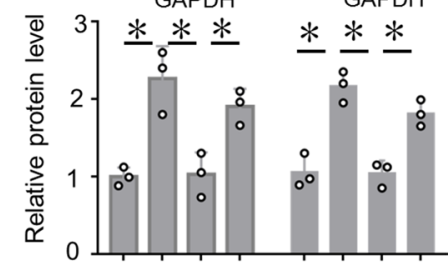

Ang II -+++-+++

SFRP2 - $-++\quad-\quad++$ 
4Fig. 3 sFRP2 overexpression prevents apoptosis in the development of cardiac hypertrophy. a Representative terminal deoxynucleotidyl transferase dUTP nick end labeling (TUNEL) images of myocardium overexpressing sFRP2 or GFP, a large number of TUNEL positive nucleus were present in the hypertrophic cardiac slices, whereas positive nucleus signal was significantly reduced in the sFRP2-overexpressing hearts. b Quantification of positive TUNEL nuclei $(n=6$ mice per group). c Protein expression of cleaved-caspase 3, BAX, and BCL-2 was analyzed by Western blot (left); quantitative results of cleaved-caspase 3 and the BAX/BCL-2 ratio (right). $\mathbf{d}$ Western blot and quantitative results of active $\beta$-catenin and total $\beta$-catenin protein in the heart of mice 4 weeks after $\mathrm{AB}$ ( $n=3$ repetitions). e NRCMs were co-incubated with $\mathrm{LiCl}(2.5 \mathrm{mM}, 12 \mathrm{~h})$ followed by stimulation with Ang II $(1 \mu \mathrm{M}, 24 \mathrm{~h})$. Representative images of cardiomyocyte hypertrophy. (blue: nuclear; green: $\alpha$-actinin; scale bars: $25 \mu \mathrm{m}$ ). f Quantitation of the NRCM cell surface area (at least 20 cells per group). g BNP mRNA expression was detected by RT-PCR analysis $(n=3$ repetitions). h Protein expression of cleaved-caspase 3 and $\beta$-catenin in NRCMs after Ang II stimulation for $24 \mathrm{~h}$. Representative Western blots (left) and quantitative data (right) are presented ( $n=3$ repetitions). Data are presented as the mean \pm SD. $* P<0.05$ vs. the sham group, $\# P<0.05$ vs. the $\mathrm{AB}+\mathrm{GFP}$ group, and $* P<0.05$, $\S P<0.05$ vs. the sham group.
Wnt-activating activity in different organ systems under multiple physiological conditions. In our opinion, there is a cardioprotective effect of sFRP2 under specific pathological circumstances.

There were some limitations and pitfalls in our study. One issue is the uncertainty of sFRP2 expression in human heart failure tissues. Future studies are warranted to evaluate human samples from patients with end-stage heart failure. Another issue is that different cell type-specific conditional overexpression of sFRP2 mice are needed to further elucidate its role in the pathogenesis of cardiac hypertrophy. Obviously, more studies are needed for future investigations.

In conclusion, our present study showed that SFRP2 expression was reduced during the progression of heart failure. Moreover, overexpression of sFRP2 by AAV9-sFRP2 infection retarded cardiac remodeling induced by pressure overload by blocking Wnt/ $\beta$-catenin signaling. Consistent with our in vivo results, $\mathrm{LiCl}$ markedly abrogated the inhibitory effects of sFRP2 on cardiomyocyte hypertrophy following Ang II stimulation. Our studies implied that inhibition
A

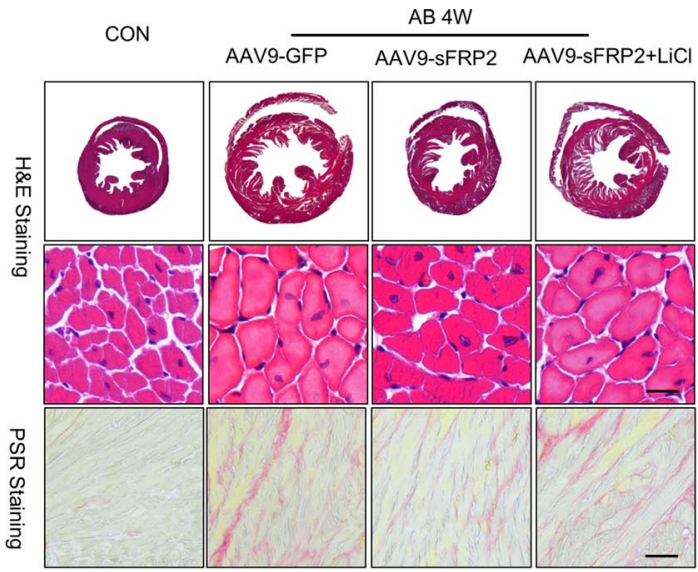

$\mathbf{F}$

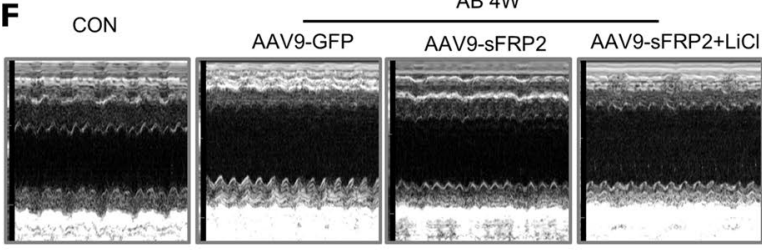

B
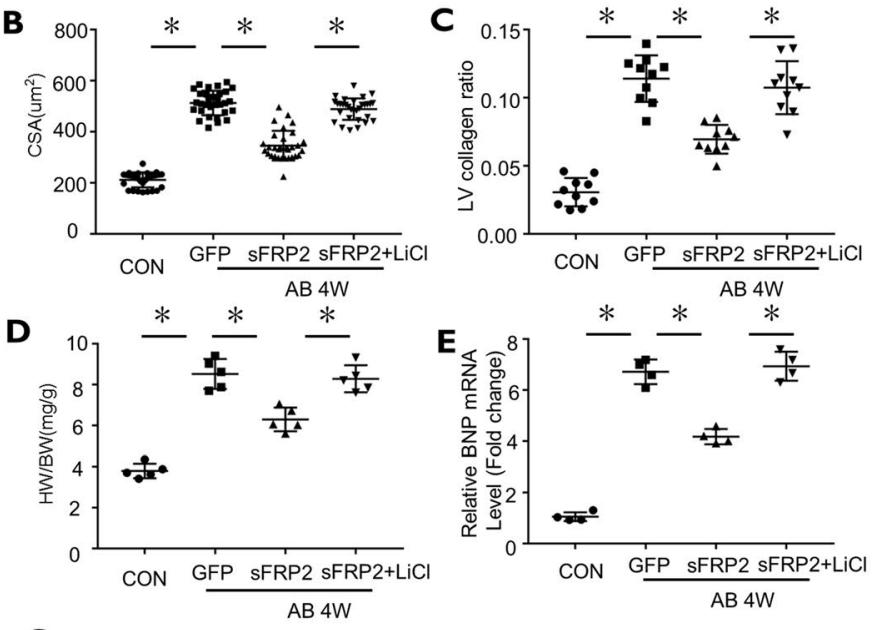

G
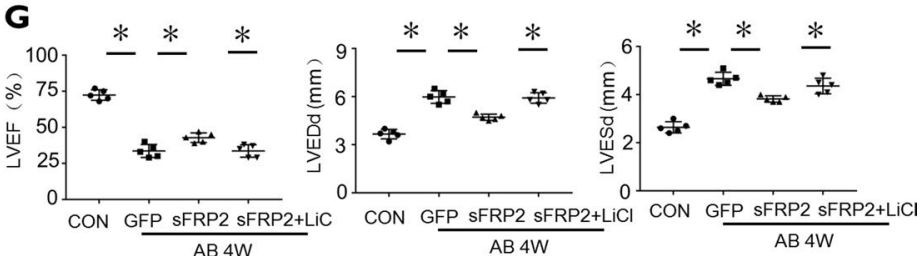

Fig. 4 sFRP2 exerted protective effects in the development of cardiac hypertrophy via inhibition of $\mathrm{Wnt} / \beta$-catenin signaling. a Representative images of the morphological analysis of cardiac hypertrophy as reflected by H\&E staining (top) and PSR staining (bottom) ( $n=5$ mice per group, at least 12 different areas were measured per group). b Cardiomyocyte cross-sectional area (CSA) quantified from the sham or $\mathrm{AB}$ mice in the indicated groups ( $n=5$ mice per group, at least 50 cells were measured per group). $\mathbf{c}$ Quantification of interstitial fibrosis from mice with sham or $\mathrm{AB}$ surgery $(n=5$ mice per group, at least 12 different areas were measured per group). d The heart weight to body weight ratio (HW/BW, $n=5$ mice per group). $\mathrm{e}$ RT-PCR analysis of BNP mRNA expression ( $n=3$ mice per group). $\mathbf{f}$ Representative echocardiographic images of each group. g Echocardiographic parameters including the left ventricle end-diastolic diameter (LVEDd), left ventricle end-systolic diameter (LVESd), and left ventricle ejection fraction (LVEF) in the indicated groups $(n=5$ mice per group). Data are presented as the mean \pm SD. $* P<0.05$ 
of $\beta$-catenin by carrying sFRP2 with AAV9 virus may be a novel strategy for protecting against cardiac remodeling in the therapy of patients with heart failure.

Acknowledgements This study was supported by grants obtained from the Youth Fund of the Affiliated Hospital of Qingdao University.

Author contributions QZ and YL performed the aortic banding, helped with the data analysis, and wrote the draft manuscript. Dr. MJW and SZW performed the Western blot and RT-PCR experiments. Dr. SZW and WZZ were involved in the experiment data analysis and pathological experiments. Dr. NZ and W-YW were responsible for the ideas and involved in the experimental design and modification and revising the manuscript.

\section{Compliance with ethical standards}

Conflict of interest The authors declare that they have no conflicts of interest.

\section{References}

1. Tanai E, Frantz S (2015) Pathophysiology of heart failure. Compr Physiol 6(1):187-214. https://doi.org/10.1002/cphy.c140055

2. Pandur P, Lasche M, Eisenberg LM, Kuhl M (2002) Wnt-11 activation of a non-canonical Wnt signalling pathway is required for cardiogenesis. Nature 418(6898):636-641. https://doi. org/10.1038/nature00921

3. Zhang Z, Deb A, Zhang Z, Pachori A, He W, Guo J, Pratt R, Dzau VJ (2009) Secreted frizzled related protein 2 protects cells from apoptosis by blocking the effect of canonical Wnt3a. J Mol Cell Cardiol 46(3):370-377. https://doi.org/10.1016/j.yjmcc .2008.11.016

4. Zhao Y, Wang C, Hong X, Miao J, Liao Y, Hou FF, Zhou L, Liu Y (2019) Wnt/beta-catenin signaling mediates both heart and kidney injury in type 2 cardiorenal syndrome. Kidney Int 95(4):815-829. https://doi.org/10.1016/j.kint.2018.11.021

5. Lin H, Angeli M, Chung KJ, Ejimadu C, Rosa AR, Lee T (2016) sFRP2 activates Wnt/beta-catenin signaling in cardiac fibroblasts: differential roles in cell growth, energy metabolism, and extracellular matrix remodeling. Am J Physiol Cell Physiol 311(5):C710 c719. https://doi.org/10.1152/ajpcell.00137.2016

6. Wei WY, Ma ZG, Zhang N, Xu SC, Yuan YP, Zeng XF, Tang QZ (2018) Overexpression of CTRP3 protects against sepsis-induced myocardial dysfunction in mice. Mol Cell Endocrinol 476:27-36. https://doi.org/10.1016/j.mce.2018.04.006

7. Song J, Zhu Y, Li J, Liu J, Gao Y, Ha T, Que L, Liu L, Zhu G, Chen Q, Xu Y, Li C, Li Y (2015) Pellino1-mediated TGFbeta1 synthesis contributes to mechanical stress induced cardiac fibroblast activation. J Mol Cell Cardiol 79:145-156. https://doi. org/10.1016/j.yjmcc.2014.11.006

8. Hulot JS, Fauconnier J, Ramanujam D, Chaanine A, Aubart F, Sassi Y, Merkle S, Cazorla O, Ouille A, Dupuis M, Hadri L, Jeong D, Muhlstedt S, Schmitt J, Braun A, Benard L, Saliba Y, Laggerbauer B, Nieswandt B, Lacampagne A, Hajjar RJ, Lompre AM, Engelhardt S (2011) Critical role for stromal interaction molecule 1 in cardiac hypertrophy. Circulation 124(7):796-805. https://doi. org/10.1161/circulationaha.111.031229

9. Duan H, Yan Z, Chen W, Wu Y, Han J, Guo H, Qiao J (2017) TET1 inhibits EMT of ovarian cancer cells through activating Wnt/beta-catenin signaling inhibitors DKK1 and SFRP2.
Gynecol Oncol 147(2):408-417. https://doi.org/10.1016/j.ygyno 2017.08.010

10. ter Horst P, Smits JF, Blankesteijn WM (2012) The Wnt/Frizzled pathway as a therapeutic target for cardiac hypertrophy: where do we stand? Acta Physiol 204(1):110-117. https://doi.org/10.11 11/j.1748-1716.2011.02309.x

11. Bovolenta P, Esteve P, Ruiz JM, Cisneros E, Lopez-Rios J (2008) Beyond Wnt inhibition: new functions of secreted Frizzled-related proteins in development and disease. J Cell Sci 121(Pt 6):737746. https://doi.org/10.1242/jcs.026096

12. Alfaro MP, Vincent A, Saraswati S, Thorne CA, Hong CC, Lee E, Young PP (2010) sFRP2 suppression of bone morphogenic protein (BMP) and Wnt signaling mediates mesenchymal stem cell (MSC) self-renewal promoting engraftment and myocardial repair. J Biol Chem 285(46):35645-35653. https://doi.org/10.1074/jbc. M110.135335

13. Mastri M, Shah Z, Hsieh K, Wang X, Wooldridge B, Martin S, Suzuki G, Lee T (2014) Secreted frizzled-related protein 2 as a target in antifibrotic therapeutic intervention. Am J Physiol Cell Physiol 306(6):C531-C539. https://doi.org/10.1152/ajpcell.00238 .2013

14. Blankesteijn WM, Essers-Janssen YP, Ulrich MM, Smits JF (1996) Increased expression of a homologue of drosophila tissue polarity gene "frizzled" in left ventricular hypertrophy in the rat, as identified by subtractive hybridization. J Mol Cell Cardiol 28(5):1187-1191

15. van de Schans VA, van den Borne SW, Strzelecka AE, Janssen BJ, van der Velden JL, Langen RC, Wynshaw-Boris A, Smits JF, Blankesteijn WM (2007) Interruption of Wnt signaling attenuates the onset of pressure overload-induced cardiac hypertrophy. Hypertension 49(3):473-480. https://doi.org/10.1161/01. Hyp.0000255946.55091.24

16. Malekar P, Hagenmueller M, Anyanwu A, Buss S, Streit MR, Weiss CS, Wolf D, Riffel J, Bauer A, Katus HA, Hardt SE (2010) Wnt signaling is critical for maladaptive cardiac hypertrophy and accelerates myocardial remodeling. Hypertension 55(4):939-945. https://doi.org/10.1161/hypertensionaha.109.141127

17. Hahn JY, Cho HJ, Bae JW, Yuk HS, Kim KI, Park KW, Koo BK, Chae IH, Shin CS, Oh BH, Choi YS, Park YB, Kim HS (2006) Beta-catenin overexpression reduces myocardial infarct size through differential effects on cardiomyocytes and cardiac fibroblasts. J Biol Chem 281(41):30979-30989. https://doi. org/10.1074/jbc.M603916200

18. Deb A, Davis BH, Guo J, Ni A, Huang J, Zhang Z, Mu H, Dzau VJ (2008) SFRP2 regulates cardiomyogenic differentiation by inhibiting a positive transcriptional autofeedback loop of Wnt3a. Stem Cells 26(1):35-44. https://doi.org/10.1634/stemcells.2007-0475

19. Mirotsou M, Zhang Z, Deb A, Zhang L, Gnecchi M, Noiseux N, Mu H, Pachori A, Dzau V (2007) Secreted frizzled related protein 2 (Sfrp2) is the key Akt-mesenchymal stem cell-released paracrine factor mediating myocardial survival and repair. Proc Natl Acad Sci USA 104(5):1643-1648. https://doi.org/10.1073/pnas.06100 24104

20. Zimmerman ZF, Kulikauskas RM, Bomsztyk K, Moon RT, Chien AJ (2013) Activation of Wnt/beta-catenin signaling increases apoptosis in melanoma cells treated with trail. PLoS ONE 8(7):e69593. https://doi.org/10.1371/journal.pone.0069593

21. Zu G, Guo J, Che N, Zhou T, Zhang X, Wang G, Ji A, Tian $X$ (2016) Protective effects of ginsenoside $\operatorname{Rg} 1$ on intestinal ischemia/reperfusion injury-induced oxidative stress and apoptosis via activation of the Wnt/beta-catenin pathway. Sci Rep 6:38480. https://doi.org/10.1038/srep38480

22. Guo Y, Gupte M, Umbarkar P, Singh AP, Sui JY, Force T, Lal H (2017) Entanglement of GSK- $3 \beta, \beta$-catenin and TGF- $\beta 1$ signaling network to regulate myocardial fibrosis. J Mol Cell Cardiol 110:109-120. https://doi.org/10.1016/j.yjmcc.2017.07.011 
23. Blyszczuk P, Muller-Edenborn B, Valenta T, Osto E, Stellato M, Behnke S, Glatz K, Basler K, Luscher TF, Distler O, Eriksson U, Kania G (2017) Transforming growth factor-beta-dependent Wnt secretion controls myofibroblast formation and myocardial fibrosis progression in experimental autoimmune myocarditis. Eur Heart J 38(18):1413-1425. https://doi.org/10.1093/eurheartj/ehw116

24. Tanaka S, Terada K, Nohno T (2011) Canonical Wnt signaling is involved in switching from cell proliferation to myogenic differentiation of mouse myoblast cells. J Mol Signaling 6:12. https ://doi.org/10.1186/1750-2187-6-12
25. He W, Zhang L, Ni A, Zhang Z, Mirotsou M, Mao L, Pratt RE, Dzau VJ (2010) Exogenously administered secreted frizzled related protein 2 (Sfrp2) reduces fibrosis and improves cardiac function in a rat model of myocardial infarction. Proc Natl Acad Sci USA 107(49):21110-21115. https://doi.org/10.1073/ pnas. 1004708107

Publisher's Note Springer Nature remains neutral with regard to jurisdictional claims in published maps and institutional affiliations. 